SLAC-TN-05-048

May 2005

\title{
Fringe Pattern of the PEP-II Synchrotron-Light Interferometers ${ }^{*}$
}

\author{
Alan Fisher \\ Stanford Linear Accelerator Center, Stanford University, Stanford, CA 94309
}

\begin{abstract}
Synchrotron-light interferometry is used to measure the vertical beam sizes in the highenergy and low-energy rings (HER and LER) of the PEP-II B Factory at SLAC. Light from a point in a dipole magnet is diffracted by two slits and then imaged onto a CCD camera. A curve fitting algorithm matches the measured interference fringes to a calculated pattern that includes the effect on the modulation depth of the fringes due to both the small but nonzero source size and the narrow bandpass of the optical filter. These formulas are derived here. Next, an additional focusing term from the primary mirror in the vacuum chamber is considered. The mirror needs extensive cooling due to the intense fan of synchrotron x-rays and is likely to have a slight stress-induced curvature, which must be considered to determine the true source size.
\end{abstract}

* Supported by U.S. Department of Energy contract DE-AC02-76SF00515. 


\section{Fringe Pattern of the PEP-II Synchrotron-Light Interferometers}

\section{A. Introduction}

Mitsuhashi first applied a two-slit interferometer for the measurement of small beam sizes at KEK a decade ago [1]. His inspiration was work done over a century earlier by Michelson, as a method to determine the diameter of a star, which still cannot be resolved directly even when viewed through a telescope. The concept is based on the theory of partial coherence-well outlined by Born and Wolf [2] - in which the fringe pattern is studied as the source size grows from a point source. The resulting differences in optical path from different points on the source lead to a loss of modulation depth of the fringes, allowing the source size to be determined from the modulation.

The formulas to be derived here thus have a long history, but they are set down here for convenience (mostly the author's), and to obtain a form that is efficient for curve fitting. In the two interferometers, on the two rings of PEP-II, a nonlinear curve fit determines the beam size by fitting the measurements to this functional form.

\section{B. Layout}

The calculation we follow is one dimensional, with two slits (Figure 1) that are narrow in $y$ and infinitely long in $x$. The slit plane is a distance $z_{0 s}$ from the source plane. A lens with focal length $f$ is a short distance $z_{s l}$ from the slits; the sum of these two, the distance from source to lens, is then $z_{0 l}$. Finally, the lens images the source plane at a distance from the lens of $z_{l i}=f+z_{f i}$.

My earlier derivation started with a single slit of narrow width $a$, then added the second slit separated by a distance $d>>a$, so that the major change in optical path, leading to partial coherence, is the path difference due to $d$. However, it is not clear that this is sufficient when the RMS beam size $\sigma$ is comparable to $a$. Instead, our procedure here will use a diffraction integral over both slits from a monochromatic point source. We then allow the source size to grow. Finally, we integrate this result over the narrow bandpass filter.

\section{Compensation for Mirror Distortion}

\section{Cylindrical Lens}

One difficulty of each of the PEP-II synchrotron-light monitors [3], including the interferometers [4], is the high heat load on the primary mirror M1 in the vacuum chamber. Thermal distortion, and mechanical distortion due to the extensive cooling and stiff water-cooling tubes attached to the back of the mirror, result in a surface that is not as flat as intended. The resulting phase variations across the surface would normally spoil the interference fringes. To remedy this, we use only two small portions of the mirror. For a vertical beam-size measurement, the apertures of the two slits themselves transmit light from only two long horizontal stripes across the mirror face. We further limit the region used by inserting a cylindrical lens downstream of the lens $f$, in order to shorten the focal length in only one plane. The camera then images the horizontal position along the mirror but the vertical position on the source plane. In the image, we still see the 
parallel stripes of the interference fringes, but the position along the stripes now corresponds to a position on the mirror face. The computer can then select a region from the image with the best fringe modulation, arising from light reflected from two small rectangular areas of the mirror. Since our calculation is one dimensional, using $y$ only, we can neglect the cylindrical lens in the diffraction calculation.

\section{2. $\quad$ Phase Offset}

These two regions are then sufficiently flat, but they may still be at a hill or valley and so have a different optical path to the midpoint of the slit plane. We address that issue by including a possible phase $\phi(v)$ as a function of position $v$ on the slit plane, but in subsequent simplification, this will be replaced by an offset $\phi_{0}$ between slits 1 and 2, which will appear in the diffraction integral (4) below.

\section{3. $\quad$ Focusing by $M 1$}

The two regions of the mirror M1 may also have a small relative tilt, leading to focusing in addition to that provided by lens $f$. This focal length is long, and so replaces the real source at the beam, a distance $z_{b m}$ from the mirror, by an effective source at the virtual image of the beam. It is this virtual source, at a distance $z_{0 m}$ from the mirror, that we consider below. Because the effective source is not at the nominal distance from the lens, the camera is mounted on a translation stage to allow focusing, and because the virtual image involves some magnification, the true beam size must be found afterward by correcting the result found by the interferometer for this additional magnification. We will return to this point in section E, after deriving the fringe pattern for our source.

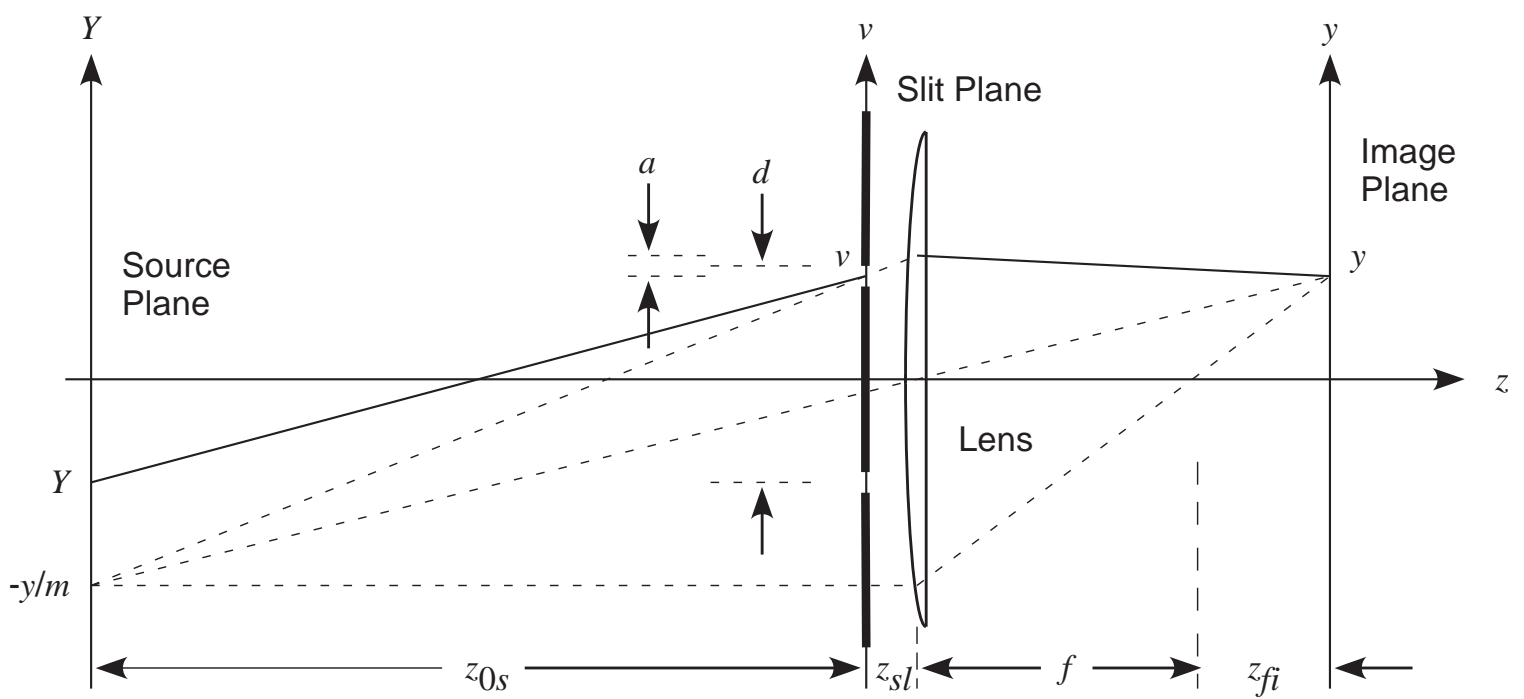

Figure 1. Geometrically imaged rays from $-y / m$ (where $m$ is the magnification) on the source plane reach their image $y$ while traveling through an equal optical path. (Of course, some of these rays are blocked by the slits.) One ray passes through the opening at $v$ in the slit plane. The ray from $Y$ reaches also reaches $y$, but by diffraction at $v$. Its optical path is equal to the others except for the initial portion $Y v$, as discussed in Section D.1. 


\section{Derivation of the Fringe Pattern}

\section{Difference in Optical Path}

Consider the arrangement of Figure 1. A ray from a point $Y$ on the $X Y$ source plane passes through the $u v$ slit plane at coordinate $v$, and reaches the $x y$ image plane at $y$. Due to diffraction at the slit, $y$ is not in general the image of the point $Y$. We need to find the extra optical path for this ray compared to the path of a non-diffracted ray-which follows ordinary geometric optics - also ending at $y$. This reference path must originate on the source plane at the image point corresponding to $y$. We can find the source-plane coordinate of this point using the magnification:

$$
-\frac{y}{m}=-\frac{z_{0 s}+z_{s l}}{z_{l i}} y=-\frac{z_{0 l}}{z_{l i}} y
$$

Recall that for geometric optics, all rays leaving the source plane at this coordinate and arriving at $y$ on the image plane have the same optical path, independent of the coordinate $v$ on the slit plane. Our diffracted ray follows the same path as this geometrically imaged ray after the slits, but has a different path before. Thus the path difference is:

$$
\begin{aligned}
\Delta s & =\sqrt{(v-Y)^{2}+z_{0 s}{ }^{2}}-\sqrt{\left(v+\frac{z_{0 l}}{z_{l i}} y\right)^{2}+z_{0 s}{ }^{2}} \\
& \approx \frac{1}{2 z_{0 s}}\left[-2 v\left(Y+\frac{z_{0 l}}{z_{l i}} y\right)+Y^{2}-\left(\frac{z_{0 l}}{z_{l i}} y\right)^{2}\right] \\
& =\frac{1}{2 z_{0 s}}\left[-2 v\left(Y+\frac{f y}{z_{f i}}\right)+Y^{2}-\left(\frac{f y}{z_{f i}}\right)^{2}\right] \\
& =-(g v+h)
\end{aligned}
$$

Here we have used the imaging relationship of the lens to substitute for the distance $z_{0 l}$ from source to lens. We do this because the distance and the focal length are known, while the effective source point for the lens_and the effective beam size-may be altered by distortion of mirror M1, as discussed above.

\section{Diffraction from a Monochromatic Point Source}

For a monochromatic source with wavenumber $k$, we can write a one-dimensional Fraunhofer diffraction integral [2] over the slits to find the electric field at $y$ due to this source at $Y$ :

$$
E(y)=\int_{\text {slits }} h(v) \exp [i k s(Y, v)+i k s(v, y)] d v
$$

This expression neglects constant factors outside the integral. The diffraction arises from the rapid variation of the phase of the exponential, in which $s(Y, v)$ and $s(v, y)$ give the optical path (including refraction in the lens) from $Y$ to $v$ and from $v$ to $y$. The function $h(v)$ represents the source amplitude, which for a point source is just $1 / r$ and so is essentially constant over the slits, since the source distance is much larger. The opening 
angle of synchrotron radiation will generally illuminate the two slits unequally, and so we retain the more general form for now. We have already computed in (2) the variation in optical path, and so, neglecting a constant phase factor, the electric field is given by:

$$
E(y)=\int_{\text {slits }} \sqrt{A(v)} \exp [i k \Delta s+i \phi(v)] d v
$$

Here $A(v)$ is the relative intensity of the synchrotron light at coordinate $v$ on the slit plane, and we also included the phase shift $\phi(v)$ due to M1 introduced in section 0 . The corresponding distribution in opening angle at the source is almost Gaussian for the visible (we use $450 \mathrm{~nm}$ ), with an RMS value of $0.5 \mathrm{mrad}$ for the HER and $0.8 \mathrm{mrad}$ for the LER, while the distance from source to slits is $12 \mathrm{~m}$ and $9 \mathrm{~m}$ for the HER and LER respectively, and so the RMS value of $A(v)$ has an RMS value of at least $6 \mathrm{~mm}$, while the typical slit width is $0.5 \mathrm{~mm}$. Thus, we can consider $A$ to be a constant $A_{1}$ and $A_{2}$ across the upper and lower slits, respectively. The integral (4) is then easily evaluated by substituting (2):

$$
\begin{aligned}
E(y, Y, k) & =\left(\sqrt{A_{1}} \int_{(d-a) / 2}^{(d+a) / 2} e^{-i k g v}+\sqrt{A_{2}} \int_{(-d-a) / 2}^{(-d+a) / 2} e^{-i k g v+i \phi_{0}}\right) e^{-i k h} \\
& =a e^{-i k h} \operatorname{sinc}\left(\frac{k g a}{2}\right)\left(\sqrt{A_{1}} e^{-i k g d / 2}+\sqrt{A_{2}} e^{i k g d / 2+i \phi_{0}}\right)
\end{aligned}
$$

The intensity is then given by $E^{*} E$ :

$$
I(y, Y, k)=a^{2} \operatorname{sinc}^{2}\left(\frac{k g a}{2}\right)\left[A_{1}+A_{2}+2 \sqrt{A_{1} A_{2}} \cos \left(k g d+\phi_{0}\right)\right]
$$

Figure 2 illustrates this result for a typical case.

\section{Diffraction from an Extended Source}

We replace the point source at $Y$ with a distributed Gaussian source centered at $Y_{0}$ :

$$
\frac{1}{\sqrt{2 \pi} \sigma_{0}} \exp \left[-\frac{1}{2}\left(\frac{Y-Y_{0}}{\sigma_{0}}\right)^{2}\right]
$$

The two motorized steering mirrors between M1 and the slits allow the light to strike the center of the slit plane at different angles

$$
\theta_{0}=-\frac{Y_{0}}{Z_{0 s}}
$$

as if we were moving the source center $Y_{0}$. We will retain $Y_{0}$ here, but in the curve fit it is more direct to use the angle.

The points on the distributed source represent different electrons radiating independently, and so incoherently. The total illumination at point $y$ is thus found by integrating the intensity (6) over the distribution (7). 
First, examine the significance of the distribution for the phase of the sinc and of the cosine. For the sinc, the phase change due to the distribution's width is:

$$
\frac{k a}{2} \frac{\sigma_{0}}{z_{0 s}}
$$

At $450 \mathrm{~nm}$, for typical values $a=0.5 \mathrm{~mm}, \sigma_{0}=0.3 \mathrm{~mm}$, and $z_{0 \mathrm{~s}}=10 \mathrm{~m}$, this change is 0.1 . On the other hand, for the cosine, with a typical value $d=5 \mathrm{~mm}$, the phase change is 20 times larger. It is then both reasonable and convenient to hold the sinc constant in the integration over the distribution, giving:

$$
\begin{aligned}
I(y, k)= & a^{2} \operatorname{sinc}^{2}\left[\frac{k a}{2 z_{0 s}}\left(Y_{0}+\frac{f y}{z_{f i}}\right)\right] \\
& \cdot\left\{A_{1}+A_{2}+\frac{2 \sqrt{A_{1} A_{2}}}{\sqrt{2 \pi} \sigma_{0}} \int \exp \left[-\frac{1}{2}\left(\frac{Y-Y_{0}}{\sigma_{0}}\right)^{2}\right] \cos \left[\frac{k d}{z_{0 s}}\left(Y+\frac{f y}{z_{f i}}\right)+\phi_{0}\right] d Y\right\} \\
= & a^{2} \operatorname{sinc}^{2}\left[\frac{k g_{0} a}{2}\right]\left\{A_{1}+A_{2}+2 \sqrt{A_{1} A_{2}} \exp \left[-\frac{1}{2}\left(\frac{k \sigma_{0} d}{z_{0 s}}\right)^{2}\right] \cos \left(k g_{0} d+\phi_{0}\right)\right\}
\end{aligned}
$$

as illustrated in Figure 3. Here we have defined:

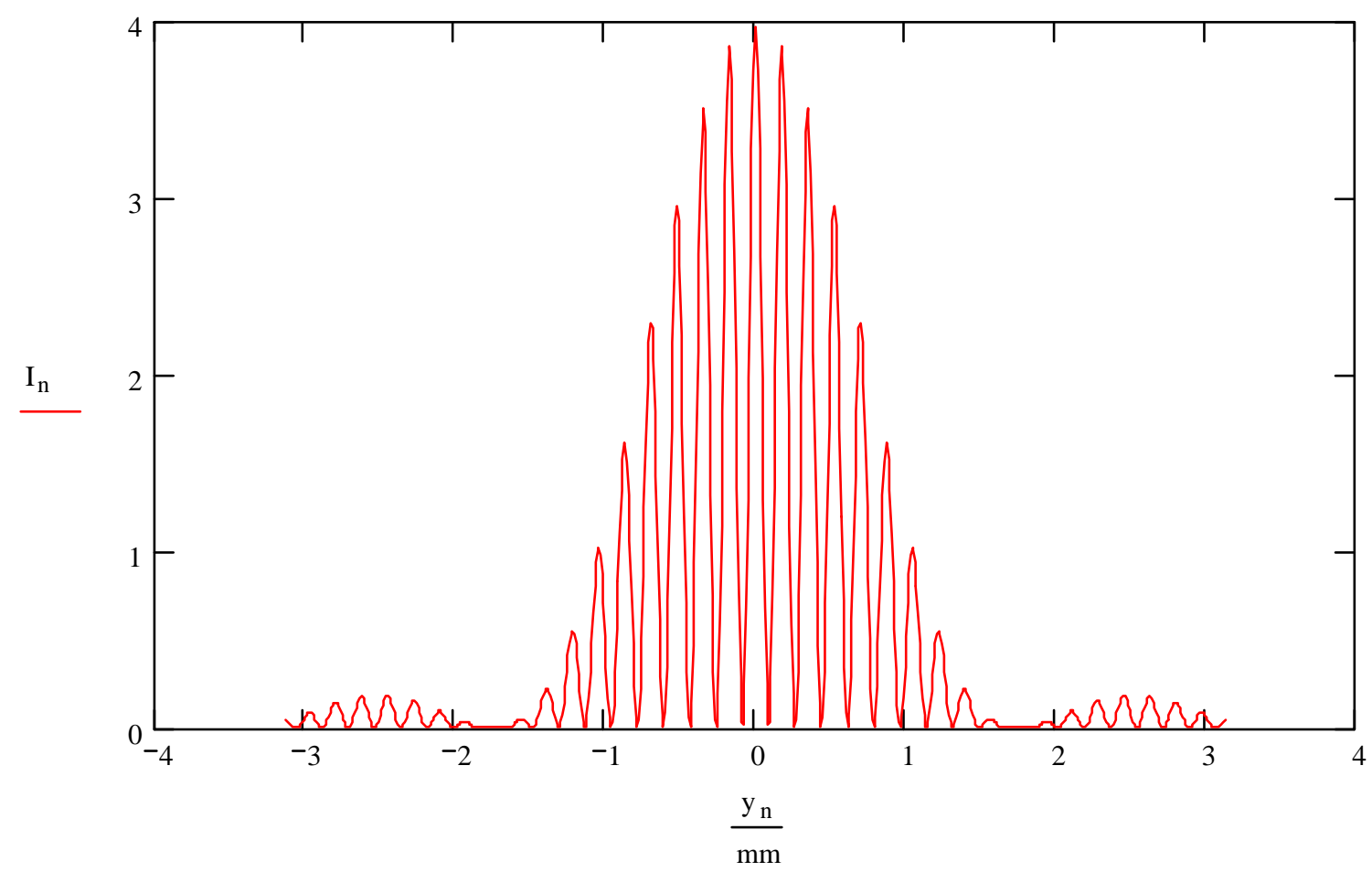

Figure 2. Fringe pattern of a monochromatic point source at $450 \mathrm{~nm}$, using parameters for the HER interferometer. $A_{1}=A_{2}=1, d=5 \mathrm{~mm}, a=0.5 \mathrm{~mm}$. 


$$
g_{0}=\frac{1}{z_{0 s}}\left(Y_{0}+\frac{f y}{z_{f i}}\right)=\frac{f y}{z_{0 s} z_{f i}}-\theta_{0}=\frac{y}{f+z_{f i}\left(1-z_{s l} / f\right)}-\theta_{0}
$$

\section{Integration over the Bandpass Filter}

We replace the monochromatic source with a narrow Gaussian band centered at wavenumber $k_{0}$ :

$$
\frac{1}{\sqrt{2 \pi} \sigma_{k}} \exp \left[-\frac{1}{2}\left(\frac{k-k_{0}}{\sigma_{k}}\right)^{2}\right]
$$

Typical values are:

$$
\begin{array}{ll}
\text { Center wavelength } & \lambda_{0}=2 \pi / k_{0}=450 \mathrm{~nm} \\
\text { Full width at half maximum } & \lambda_{\text {FWHM }}=30 \mathrm{~nm} \\
\text { RMS wavelength } & \sigma_{\lambda}=\frac{\lambda_{\text {FWHM }}}{\sqrt{8 \ln 2}}=\frac{\lambda_{\text {FWHM }}}{2.355}=12.7 \mathrm{~nm} \\
\text { RMS wavenumber } & \sigma_{k}=k_{0} \frac{\sigma_{\lambda}}{\lambda_{0}}
\end{array}
$$

Again we examine the significance of the distribution on the arguments of the sinc and cosine. For the sinc, we evaluate the phase change due to the distribution's width at the

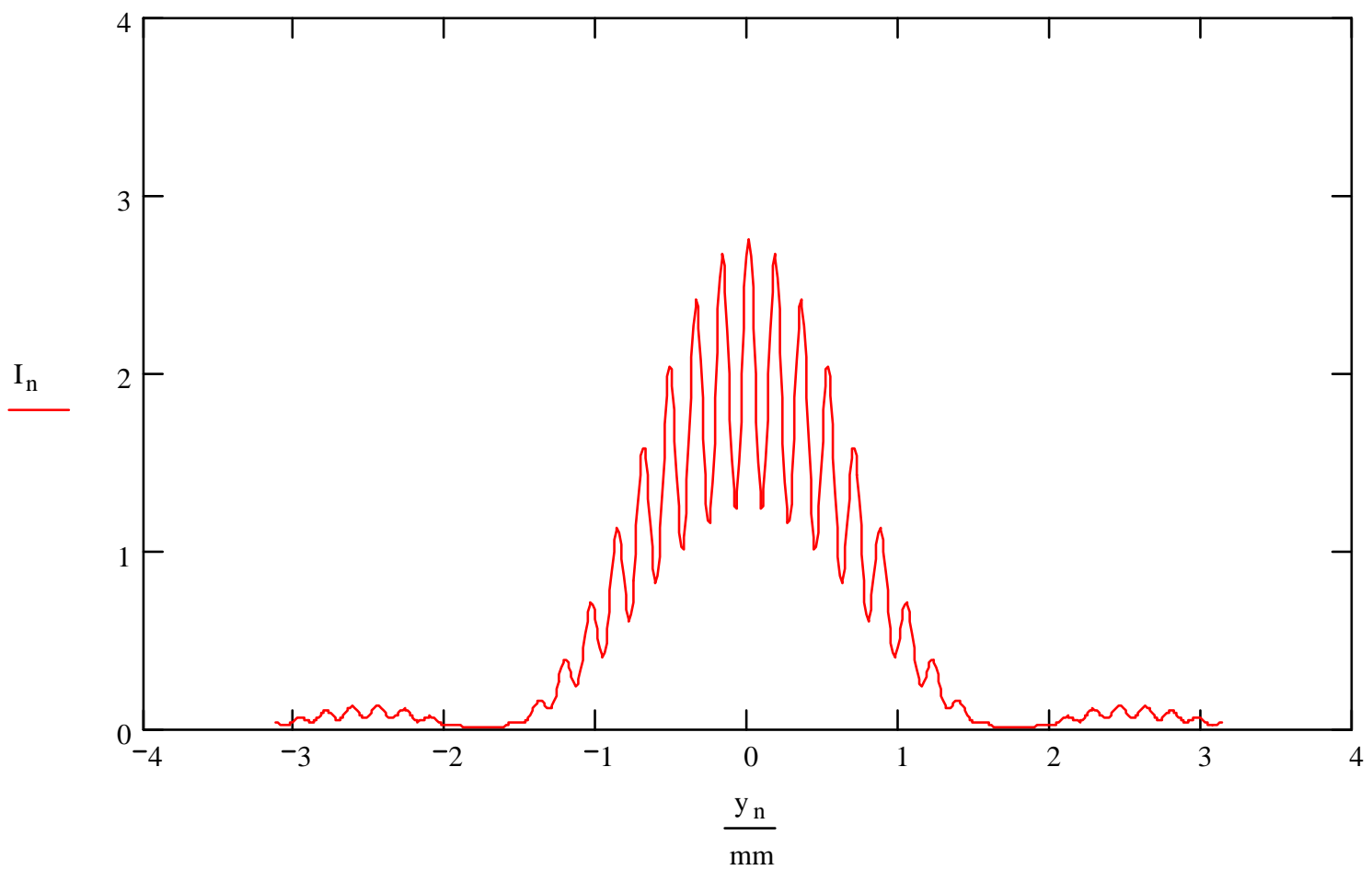

Figure 3. Fringe pattern for an extended source. Same as previous figure, but with $\sigma_{0}=0.2 \mathrm{~mm}$. 
first zero, where the argument is $\pi$, giving:

$$
\pi \frac{\sigma_{k}}{k_{0}}=0.09
$$

Again, the phase change of the cosine is 20 times larger, and so we can remove the sinc from the integral while integrating over the cosine. The exponential stays inside the integral, giving:

$$
\begin{aligned}
I(y)= & a^{2} \operatorname{sinc}^{2}\left[\frac{k_{0} g_{0} a}{2}\right] \\
& \cdot\left\{A_{1}+A_{2}+\frac{2 \sqrt{A_{1} A_{2}}}{\sqrt{2 \pi} \sigma_{k}} \int \exp \left[-\frac{1}{2}\left(\frac{k-k_{0}}{\sigma_{k}}\right)^{2}\right] \exp \left[-\frac{1}{2}\left(\frac{k \sigma_{0} d}{z_{0 s}}\right)^{2}\right] \cos \left(k g_{0} d+\phi_{0}\right) d k\right\} \\
= & a^{2} \operatorname{sinc}^{2}\left[\frac{k_{0} g_{0} a}{2}\right]\left\{A_{1}+A_{2}\right. \\
& \left.+\frac{2 \sqrt{A_{1} A_{2}}}{\sqrt{1+\left(\frac{\sigma_{k} \sigma_{0} d}{z_{0 s}}\right)^{2}}} \exp \left[-\frac{\left(\frac{k_{0} \sigma_{0} d}{z_{0 s}}\right)^{2}+\left(\sigma_{k} g_{0} d\right)^{2}}{2\left[1+\left(\frac{\sigma_{k} \sigma_{0} d}{z_{0 s}}\right)^{2}\right]}\right] \cos \left[\frac{k_{0} g_{0} d}{1+\left(\frac{\sigma_{k} \sigma_{0} d}{z_{0 s}}\right)^{2}}+\phi_{0}\right]\right\}
\end{aligned}
$$

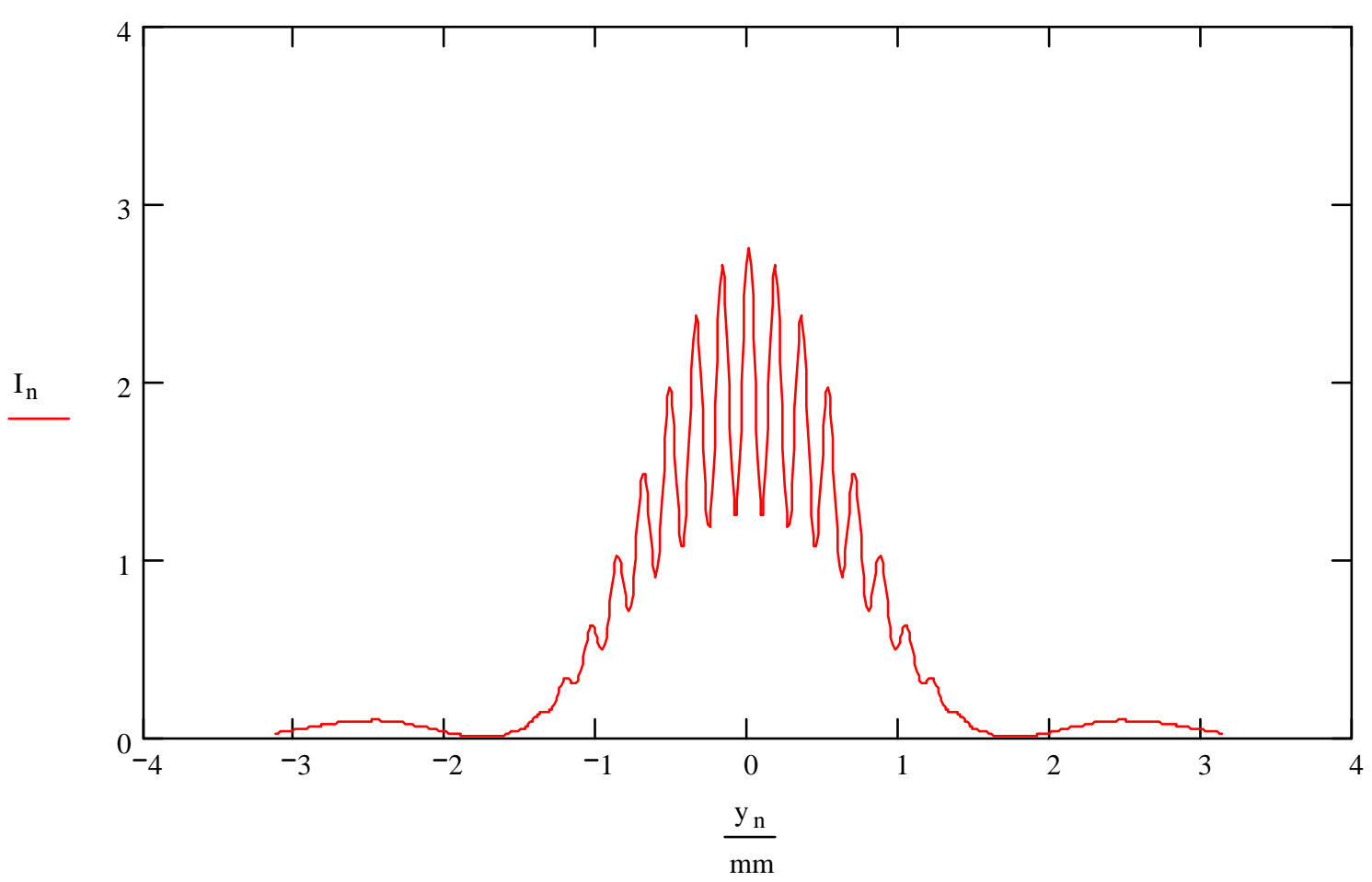

Figure 4. Extended source with a narrow bandpass. Same parameters as previous figure, but with a Gaussian band having a 30-nm full width at half maximum. 
This expression, illustrated with various parameters in Figure 4 through Figure 6, reverts to the monochromatic one (10) for zero bandwidth. As the band widens, the depth of fringe modulation decreases and the spacing of the fringes broadens slightly.

\section{Fringe Formula Used for Curve Fitting}

Before doing the fit, the measured fringe pattern is normalized to give a peak value of 1 . Consequently, in the fitting formula, we remove the leading factor of $a^{2}$. The ratio

$$
\alpha=A_{2} / A_{1}
$$

is measured by blocking one slit at a time, and is then held constant through the fit. $A_{1}$ can then be factored out and used for an overall amplitude. Finally, we add a baseline offset $b$ to account for any background illumination or pedestal at the camera. The resulting formula used in the algorithm is:

$$
\begin{aligned}
I(y)= & +A_{1} \operatorname{sinc}^{2}\left[\frac{k_{0} g_{0} a}{2}\right]\{1+\alpha \\
& +\frac{2 \sqrt{\alpha}}{\sqrt{1+\left(\frac{\sigma_{k} \sigma_{0} d}{z_{0 s}}\right)^{2}}} \exp \left[-\frac{\left(\frac{k_{0} \sigma_{0} d}{z_{0 s}}\right)^{2}+\left(\sigma_{k} g_{0} d\right)^{2}}{2\left[1+\left(\frac{\sigma_{k} \sigma_{0} d}{z_{0 s}}\right)^{2}\right]}\right] \cos \left[\frac{k_{0} g_{0} d}{1+\left(\frac{\sigma_{k} \sigma_{0} d}{z_{0 s}}\right)^{2}}+\phi_{0}\right]
\end{aligned}
$$

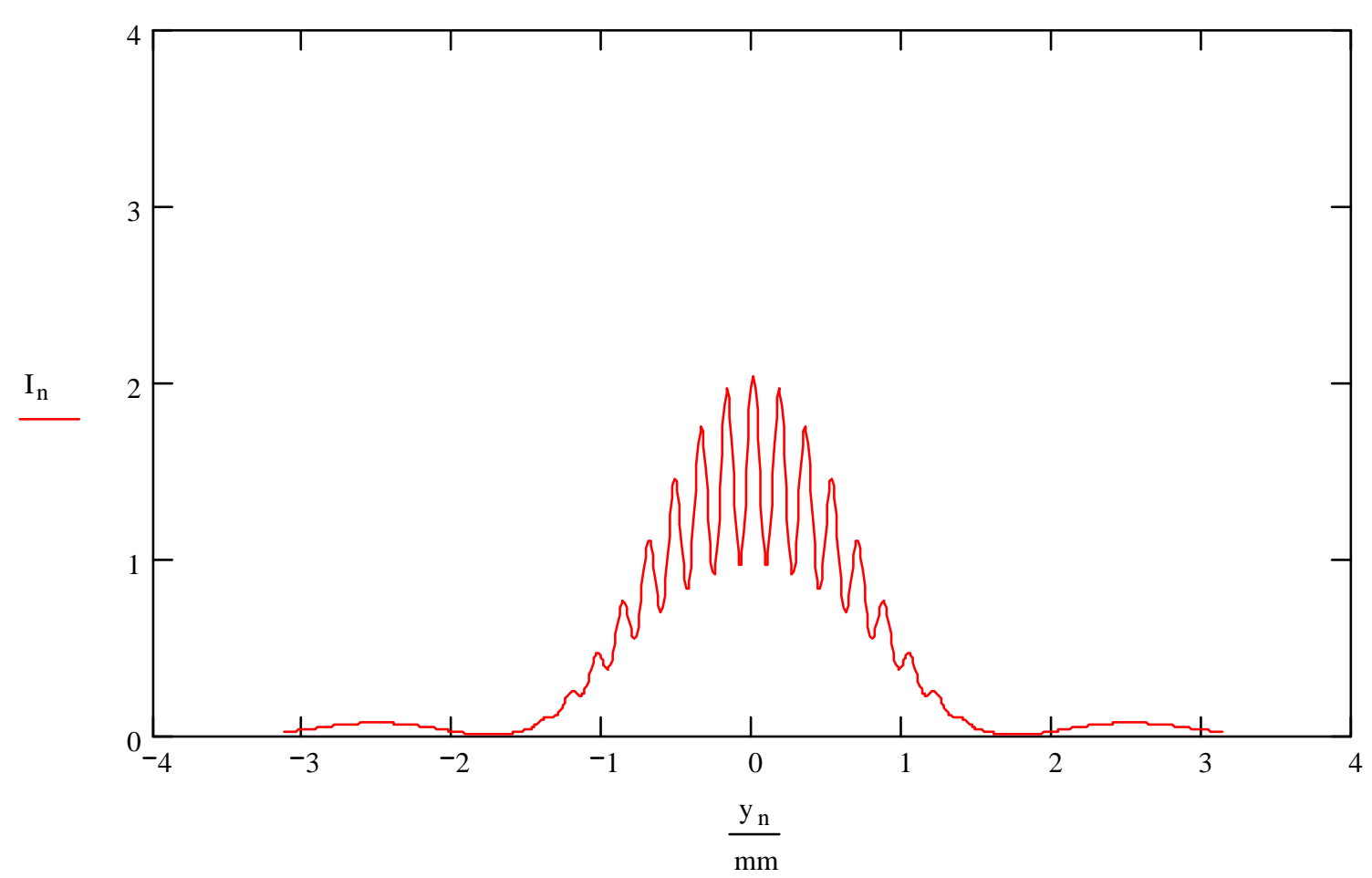

Figure 5. Same as previous figure, but the emission pattern is not centered on the slits: $A_{1}=1, A_{2}=0.5$. This case is typical, because the slot in M1 prevents illumination of the slits when the pattern is centered. 


\section{E. When the Camera is not at the Nominal Focus}

Two effects can move the effective source point away from the nominal beam source point, at a distance $z_{b s}$ upstream of the slit plane: focusing due to some curvature of M1, and a nonzero horizontal orbit offset or orbit angle that then changes the tangent striking the mirror. Which is the major reason that we sometimes move the camera, and is compensation needed in determining the beam size?

\section{Compensation for Focusing by M1}

As noted before, we use only two small regions of mirror M1. We already allowed for the possibility that they may have a different depth from the ideal flat surface, by including the arbitrary phase offset $\phi_{0}$. However, these regions can also have a relative tilt, which introduces a focusing effect. Since the focal length $f_{m}$ will be long, the mirror images the true source to a virtual source either closer to or further from the mirror, depending on the sign of the curvature.

We know the positions of the true source point, mirror, the slits, the lens, and the camera. If we assume that the user has adjusted the camera for best focus-meaning the best overlap of the slit patterns from both of the two slits-and if we know the focal length of the lens at the color passed by our filter, then the lens equation gives us the location of the effective source. Note that the expressions (18) and (11) for the fringe pattern use only the distances downstream of the slits and this focusing assumption.

The cameras in both rings have been focused to a point $60 \mathrm{~mm}$ further from the lens than the nominal focus, so that

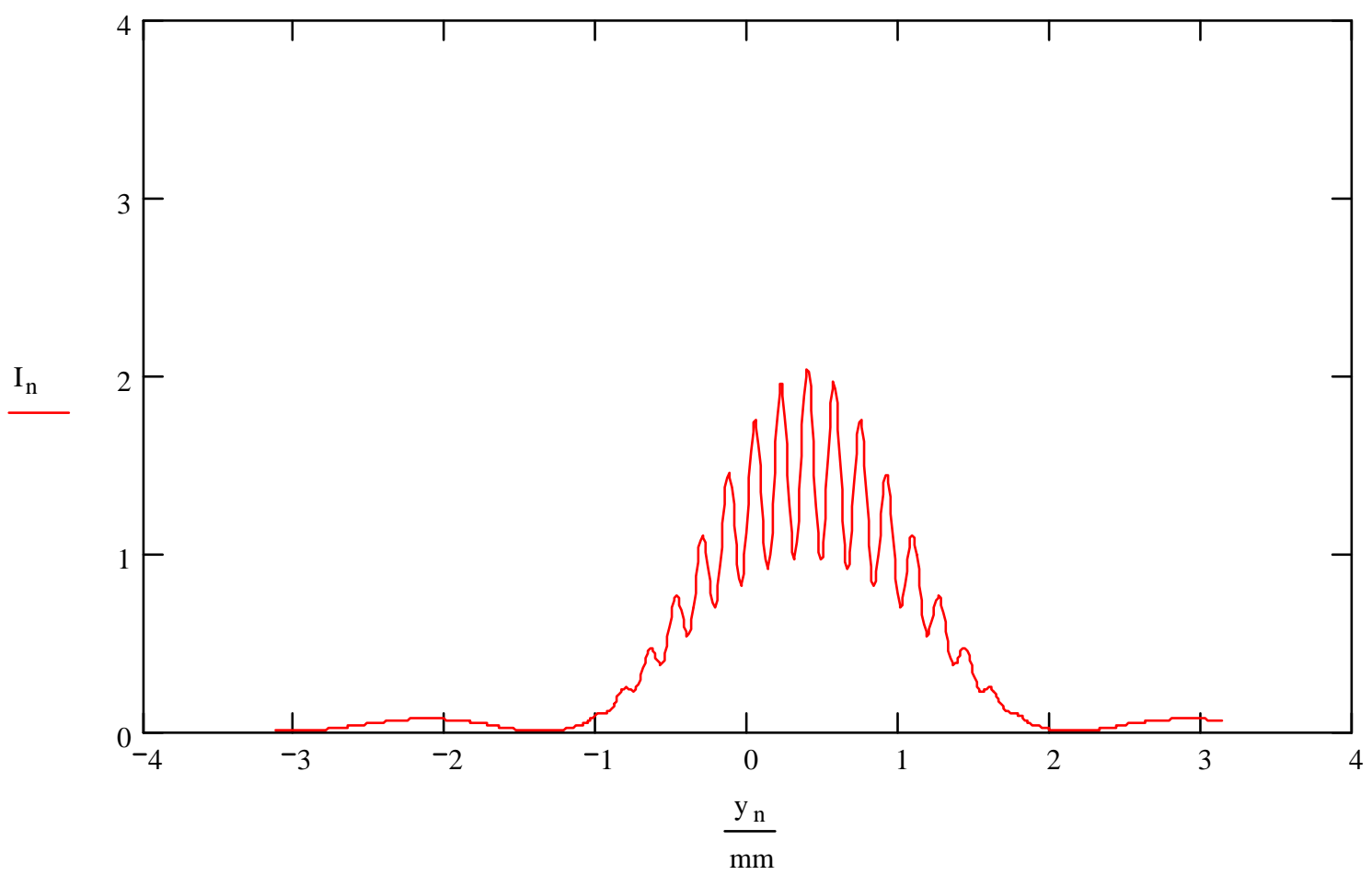

Figure 6. Same as previous figure, but the light hits the slits at a slight angle $\theta_{0}=0.2 \mathrm{mrad}$. 


$$
z_{l i}=z_{l i, \text { nom }}+z_{\text {cam }}
$$

The effective source is at

$$
z_{0 l}=\left(\frac{1}{f}-\frac{1}{z_{l i}}\right)^{-1}
$$

If we call the distance from the mirror to the slit plane $z_{m s}$, then the magnification of the true source size by M1 is

$$
M_{m}=\frac{Z_{0 s}-z_{m s}}{Z_{b s}-z_{m s}}
$$

and the focal length of M1 is

$$
f_{m}=\left(\frac{1}{z_{b s}-z_{m s}}-\frac{1}{z_{0 s}-z_{m s}}\right)^{-1}
$$

(The sign of the second term is negative because the distances are positive but the image is virtual.) For our 60-mm camera offset, the effect is significant, with a virtual source that is both closer to the mirror - by $195 \mathrm{~cm}$ for the HER and by $105 \mathrm{~cm}$ for the LERand smaller than the true source-with a magnification of 0.64 for the HER and 0.68 for the LER. The focal length for M1 is $-10 \mathrm{~m}$ for the HER and $-6.8 \mathrm{~m}$ for the LER.

This result should be used with caution, since it is difficult to precisely focus the camera. The technique involves blocking one slit at a time and finding the camera position at which the two single-slit patterns have their best overlap. The curvature is likely to vary with horizontal position along the mirror and perhaps with mirror heating, and so the focus would need to be confirmed for each location of the region of interest selected by the user from the video image and for different beam currents.

\section{Focusing Changes due to Orbit Offsets}

The alternative explanation for moving the camera from its nominal focus is that the beam's orbit is not on axis or its angle is nonzero. In this case, the size at the effective source point is the true beam size, requiring no additional correction for magnification.

For a bending angle $\alpha$ away from the nominal emission point, and for a given off-axis position $x_{\alpha}$ and orbit angle $\theta_{\alpha}$, we can evaluate the value of $\alpha$ that points the light to the middle of mirror M1. From this $\alpha$, we can compute the path difference and see if it is enough to account for the location of the effective source. We limit the typical orbit to 1 $\mathrm{mm}$, and the angle to $1 \mathrm{mrad}$.

The condition that the ray starting at $\alpha, x_{\alpha}$ and $\theta_{\alpha}$ strikes the mirror on center is:

$$
\left(\rho+x_{\alpha}\right) \cos \alpha-\rho+\left[z_{b m}-\left(\rho+x_{\alpha}\right) \sin \alpha\right] \tan \left(\theta_{\alpha}-\alpha\right)=0
$$

Here $\rho$ is the radius of curvature of the bend at the synchrotron source, $165 \mathrm{~m}$ for the HER and $43.454 \mathrm{~m}$ for the LER. Expanding to $2^{\text {nd }}$ order in the small quantities $\alpha, x_{\alpha}$ and 
$\theta_{\alpha}$, we get a quadratic in $\alpha$. Only the negative root gives $\alpha=0$ when the orbit is on axis, and so the extra optical path for this source point is

$$
-\rho \alpha \approx-\rho\left(\theta_{\alpha}+\frac{x_{\alpha}}{z_{b m}}\right)
$$

For the typical offsets of $1 \mathrm{~mm}$ and $1 \mathrm{mrad}$, this value is $-19 \mathrm{~cm}$ for the HER and $-5.7 \mathrm{~cm}$ for the LER, too small to account for the $60 \mathrm{~cm}$ offset of the camera. We thus conclude that the shape of M1 is largely the cause of the camera's position, and a correction of the beam size is indicated, to the extent that we are sure of the optimal focusing of the camera.

However, if we are to correct for the magnification due to M1, we should properly include both effects. We need to verify the focus, then use BPM readings to get the correct location of the beam's source point, and finally use this value to find the magnification.

\section{F. References}

[1] T. Mitsuhashi, "Beam Profile and Size Measurement by SR Interferometers," in Beam Measurement, Proc. Joint US-CERN-Japan-Russia School on Particle Accelerators, Montreux, Switzerland, 11-20 May 1998 (World Scientific, Singapore), pp. 399-427.

[2] M. Born and E. Wolf, Principles of Optics, $5^{\text {th }}$ ed. (Pergamon Press, Oxford, 1975).

[3] Fisher, A.S., "Instrumentation and Diagnostics for PEP-II," Proc. Beam Instrumentation Workshop, Stanford, CA, 4-7 May 1998, AIP Conf. Proc. 451 (Amer. Inst. Phys., Woodbury, NY, 1998), pp. 395-403.

[4] A.S. Fisher, E.L. Bong, R.L. Holtzapple, and M. Petree, "Beam-Size Measurements on PEP-II Using Synchrotron-Light Interferometry,” Proc. Particle Accelerator Conf., Chicago, IL, June 18-22, 2001, edited by P. Lucas and S. Webber, IEEE, Piscataway, NJ, 2001, pp. 547-549, SLAC-PUB-8867. 